\title{
Singular Minimal Translation Surfaces in Euclidean Spaces Endowed with Semi-symmetric Connections
}

\author{
Muhittin Evren Aydin ${ }^{1}$ iD, Ayla Erdur Kara ${ }^{2 * *}$ iD, Mahmut Ergut $^{3}$ iD \\ ${ }^{1}$ Department of Mathematics, Faculty of Science, Firat University, 23200, Elazig, Turkey. \\ ${ }^{2,3}$ Department of Mathematics, Faculty of Science and Art, Tekirdag Namik Kemal University, 59030, Tekirdag, Turkey.
}

Received: 19-02-2021 • Accepted: 16-09-2021

AвSTRACT. In this paper, we study and classify singular minimal translation surfaces in a Euclidean space of dimension 3 endowed with a certain semi-symmetric (non-)metric connection.

2010 AMS Classification: 53A10, 53C42, 53C05

Keywords: Singular minimal surface, translation surface, $\alpha$-catenary, semi-symmetric connection.

\section{INTRODUCTION}

Let $\mathbb{R}^{3}$ denote a Euclidean space of dimension 3 with the canonical metric $\langle$,$\rangle and (x, y, z)$ the rectangular coordinates of $\mathbb{R}^{3}$. A surface $M$ in $\mathbb{R}^{3}$ is called translation surface if it can be written as the sum of two curves. Then, a local parametrization $\sigma$ on $M$ follows $\sigma\left(s_{1}, s_{2}\right)=\gamma_{1}\left(s_{1}\right)+\gamma_{2}\left(s_{2}\right)$, for $\gamma_{i}: I_{i} \subset \mathbb{R} \rightarrow \mathbb{R}^{3}, i=1,2$, [8]. In the special case that the curves $\gamma_{i}$ lie in orthogonal planes, up to a change of coordinates, the surface $M$ can be locally expressed by the explicit form $z=f(x)+g(y)$ for smooth functions $f, g$ of single variable. If such a surface is minimal, i.e. its mean curvature vanishes identically, then it is either a plane or describes the Scherk surface [48].

While this sort of surfaces has been studied in classical manner since the former half of nineteenth century (see $[5,12-14,17,25-33,36,40,41,48-51,53-56,59,60])$, their complete classification/characterization in $\mathbb{R}^{3}$ emposing natural curvature conditions (e.g. minimality, flatness and having nonzero constant mean or Gaussian curvatures) have been recently found, see [20-22]. Yet, in higher dimensions and different ambient spaces, there are still numerous unsolved problems.

On the other hand, a semi-symmetric metric (resp. non-metric) connection on a Riemannian manifold was defined by Hayden [23] (resp. Agashe [1]) and since then has been studied by many authors. For example, see [2,3,7,9, 15, 18, $24,42-45,52,57,58,61]$.

As can be seen from the great number of published studies, the notions of translation surfaces and semi-symmetric (non-) metric connection have great interest and very recently Wang [54] combined these seperate research areas into one, which came up with a new perspective. In the cited paper, the author introduced and obtained minimal translation surfaces in 3-dimensional space forms endowed with a certain semi-symmetric (non-)metric connection.

In this study we mainly concern with singular minimal surfaces in $\mathbb{R}^{3}$, namely those surfaces satisfying an equation of mean curvature type (see [11]). The notion of singular minimal surface is a generalization of two-dimensional analogue of the catenary which is known as a model for the surfaces with the lowest gravity center, in other words, one has

*Corresponding Author

Email addresses: meaydin@ firat.edu.tr (M.E. Aydin), aerdur@nku.edu.tr (A. Erdur Kara), mergut@nku.edu.tr (M. Ergut) 
minimal potential energy [6]. In this context, the present study aims to contribute to Wang's perspective by considering singular minimal translation surfaces in $\mathbb{R}^{3}$ endowed with a certain semi-symmetric (non-)metric connection.

In order to explicitly initiate the notion of singular minimality, we begin with the one-dimensional case: let $\gamma: I \subset$ $\mathbb{R} \rightarrow \mathbb{R}^{2}$ be a parametrized curve and $\mathbf{u} \in \mathbb{R}^{2}$ a fixed unit vector and $\alpha$ some real constant. Then the curve $\gamma$ is called $\alpha$-catenary (see [11]) if the following holds

$$
\kappa(s)=\alpha \frac{\langle\mathbf{n}(s), \mathbf{u}\rangle}{\langle\gamma(s), \mathbf{u}\rangle},
$$

where $\kappa$ and $\mathbf{n}$ are the curvature and principle unit normal vector field of $\gamma$. Up to a change of coordinates, one can assumed as $\mathbf{u}=(0,1)$ and $\gamma$ a graph locally given by $\gamma(s)=(s, f(s))$, for $f: I \subset \mathbb{R} \rightarrow \mathbb{R}^{+}$. Thereby, for $\alpha=1$, Eq. (1.1) writes

$$
\frac{f^{\prime \prime}}{1+\left(f^{\prime}\right)^{2}}=\frac{1}{f}
$$

for each $s \in I$. The solution of Eq. (1.2) is the catenary $f(s)=\frac{1}{\lambda} \cosh (\lambda s+\mu), \lambda, \mu \in \mathbb{R}, \lambda \neq 0$, see [39].

As its two-dimensional analogue, Eq. (1.2) has a remarkable a physical point of view, which can be initiated as follows: let the direction of gravity be choosen as $y$-axis. Then Eq. (1.2) defines a configuration in which a uniform chain, whose two ends are fixed and hanged under its own weight, is in balance with the effect of the gravitational field. So, a catenary actually minimizes potential energy under the influence of gravity force, in other words has the lowest center of gravity (e.g. [16]).

Let us now consider the smooth immersion $\sigma: M \rightarrow \mathbb{R}_{+}^{3}(\mathbf{u})$ of an oriented surface $M$ in the halfspace

$$
\mathbb{R}_{+}^{3}(\mathbf{u})=\left\{p \in \mathbb{R}^{3},\langle p, \mathbf{u}\rangle>0\right\},
$$

for a fixed unit vector $\mathbf{u} \in \mathbb{R}^{3}$. Then, the potential $\alpha$-energy of $\sigma$ in the direction of $\mathbf{u}$ is defined by $([37,38])$

$$
E(\sigma)=\int_{M}\langle\sigma(q), u\rangle^{\alpha} d M, q \in M,
$$

where $d M$ refers to the measure on $M$ with respect to the induced metric tensor from $\mathbb{R}^{3}$. If $\sigma$ is a critical point of Eq. (1.3), it then follows

$$
2 H=\alpha \frac{\langle\xi, \mathbf{u}\rangle}{\langle\sigma, \mathbf{u}\rangle},
$$

where $H$ and $\xi$ are the mean curvature and unit normal vector field on $M$. A surface in $\mathbb{R}^{3}$ fulfilling Eq. (1.4) is called singular minimal surface or $\alpha$-minimal surface see [10,11].

Eq. (1.4) is clearly an equation of mean curvature type and reduces to the classical minimal surface equation when $\alpha=0$ [34, p. 17]. If we take $\mathbf{u}=(0,0,1)$ and $\alpha=1$ in Eq. (1.4), then the surface $M$ is said to be two-dimensional analogue of the catenary [6].

López [37] proved that a singular minimal translation surface in $\mathbb{R}^{3}$ with respect to a horizontal or a vertical direction is a $\alpha$-catenary cylinder, namely a generalized cylinder (see [19, p. 439]) whose base curve is a $\alpha$-catenary. This result was generalized to higher dimensions by the present authors [4].

Noting that the mean curvature $H$ in Eq. (1.4) is given with respect to the Levi-Civita connection on $\mathbb{R}^{3}$, we modify it by considering the mean curvatures arising via special semi-symmetric metric and non-metric connections $\nabla$ and $D$ given by Eqs. (2.1) and (2.2). We call the modified concepts $\nabla$ - and $D$-singular minimality and these allow us non-trivial and new problems. One of the problems is to find $\nabla$ - and $D$-singular minimal translation surfaces with respect to a horizontal direction and we solve it completely.

\section{Preliminaries}

Let $(\tilde{M}, g)$ be a Riemannian manifold of dimension 3 and $\tilde{\nabla}$ an affine connection on $\tilde{M}$. Let us denote the set of sections of a vector bundle $E \rightarrow \tilde{M}$ by $\Gamma(E)$ and the set of tensor fields of type $(r, s)$ on $\tilde{M}$ by $\Gamma\left(T \tilde{M}^{(r, s)}\right)$. Then the torsion tensor field $T \in \Gamma\left(T \tilde{M}^{(1,2)}\right)$ of $\tilde{\nabla}$ is defined by

$$
T(X, Y)=\tilde{\nabla}_{X} Y-\tilde{\nabla}_{Y} X-[X, Y]
$$

for $X, Y \in \Gamma(T \tilde{M})$. Then the connection $\tilde{\nabla}$ is called (see [42,58])

(1) a (resp. non-) symmetric connection if $T$ (resp. does not) vanishes identically; 
(2) a (resp. non-) metric connection if $g$ is (resp. not) parallel;

(3) a semi-symmetric connection if the following holds

$$
T(X, Y)=\pi(Y) X-\pi(X) Y,
$$

for $\pi(X)=g(X, W), \pi \in \Gamma\left(T \tilde{M}^{(0,1)}\right), W \in \Gamma(T \tilde{M}) ;$

(4) a Levi-Civita connection if it is both symmetric and metric.

Let $\tilde{M}=\mathbb{R}^{3}$ and $\left\{\partial_{x}, \partial_{y}, \partial_{z}\right\}$ the standard basis on $\mathbb{R}^{3}$. Consider the certain semi-symmetric metric and non-metric connections, respectively [54]

$$
\nabla_{X} Y=\nabla_{X}^{L} Y+g\left(Y, \partial_{z}\right) X-g(X, Y) \partial_{z}
$$

and

$$
D_{X} Y=\nabla_{X}^{L} Y+g\left(Y, \partial_{z}\right) X
$$

where $\nabla^{L}$ is the Levi-Civita connection on $\mathbb{R}^{3}$ and $X, Y \in \Gamma\left(T \mathbb{R}^{3}\right)$. For Eqs. (2.1) and (2.2), the nonzero derivatives are given by

$$
\nabla_{\partial_{x}} \partial_{x}=-\partial_{z}, \nabla_{\partial_{x}} \partial_{z}=\partial_{x}, \nabla_{\partial_{y}} \partial_{y}=-\partial_{z}, \nabla_{\partial_{y}} \partial_{z}=\partial_{y},
$$

and

$$
D_{\partial_{x}} \partial_{z}=\partial_{x}, D_{\partial_{y}} \partial_{z}=\partial_{y}, D_{\partial_{z}} \partial_{z}=\partial_{z}
$$

Let $M$ be an oriented immersed surface into $\mathbb{R}^{3}$. For any $X, Y \in \Gamma\left(T \mathbb{R}^{3}\right)$ and $\xi \in \Gamma\left(T \mathbb{R}^{3^{\perp}}\right)$, the Gauss formulae with respect to $\nabla$ and $D$ follow

$$
\nabla_{X} Y=\left(\nabla_{X} Y\right)^{\top}+h^{\nabla}(X, Y) \xi
$$

and

$$
D_{X} Y=\left(\nabla_{X} Y\right)^{\top}+h^{D}(X, Y) \xi
$$

where $\mathrm{T}$ is the projection on the tangent bundle of $M$ and $h^{\nabla}$ and $h^{D}$ are so-called the second fundamental forms with respect to $\nabla$ and $D$, respectively. Let $\left\{e_{1}, e_{2}\right\}$ be an orthonormal tangent frame on $M$. Then the mean curvatures of $M$ with respect to $\nabla$ and $D$ are defined by

$$
H^{\nabla}=\frac{1}{2}\left[h^{\nabla}\left(e_{1}, e_{1}\right)+h^{\nabla}\left(e_{2}, e_{2}\right)\right]
$$

and

$$
H^{D}=\frac{1}{2}\left[h^{D}\left(e_{1}, e_{1}\right)+h^{D}\left(e_{2}, e_{2}\right)\right] .
$$

The surface $M$ is said to be minimal with respect to $\nabla$ (resp. $D$ ) if $H^{\nabla}$ (resp. $H^{D}$ ) vanishes.

Let $g_{i j}, 1 \leq i, j \leq 2$, denote the components of the induced metric tensor on $M$ from the canonical metric. Then the mean curvatures $H^{\nabla}$ and $H^{D}$ are respectively given by

$$
H^{\nabla}=\frac{g_{22} h_{11}^{\nabla}-g_{12}\left(h_{12}^{\nabla}+h_{21}^{\nabla}\right)+g_{11} h_{22}^{\nabla}}{2 \operatorname{det} g_{i j}}
$$

and

$$
H^{D}=\frac{g_{22} h_{11}^{D}-g_{12}\left(h_{12}^{D}+h_{21}^{D}\right)+g_{11} h_{22}^{D}}{2 \operatorname{det} g_{i j}},
$$

where $h_{i j}^{\nabla}=\left\langle\nabla_{e_{i}} e_{j}, \xi\right\rangle$ and $h_{i j}^{D}=\left\langle D_{e_{i}} e_{j}, \xi\right\rangle$, for some basis $\left\{e_{1}, e_{2}\right\}$ of $\Gamma(T M)$.

We utterly enable to introduce the notion of singular minimality with respect to the connections $\nabla$ and $D$ : let $\sigma: M \rightarrow \mathbb{R}^{3}$ be a smooth immersion of an oriented surface $M$ and $\mathbf{u} \in \mathbb{R}^{3}$ a fixed unit vector. The surface $M$ is called $\nabla$-singular minimal surface with respect to the vector $\mathbf{u}$ if it holds

$$
2 H^{\nabla}=\alpha \frac{\langle\xi, \mathbf{u}\rangle}{\langle\sigma, \mathbf{u}\rangle},\langle\sigma, \mathbf{u}\rangle \neq 0
$$

where $\xi$ is the unit normal vector field on $M$ and $\alpha$ a real constant. Accordingly the surface $M$ is called $D-$ singular minimal surface with respect to the vector $\mathbf{u}$ if it holds

$$
2 H^{D}=\alpha \frac{\langle\xi, \mathbf{u}\rangle}{\langle\sigma, \mathbf{u}\rangle},\langle\sigma, \mathbf{u}\rangle \neq 0 .
$$


It is obvious that these notions coincide with the usual minimality with respect to $\nabla$ and $D$ when $\alpha=0$ and so $\alpha \neq 0$ is assumed throughout the study.

\section{3. $\nabla$-singular Minimal Translation Surfaces}

In this section, we characterize $\nabla$-singular minimal translation surfaces in $\mathbb{R}^{3}$. As the translation property of the surface changes, Eq. (2.3) generates different tasks. Because there are three types of translation surfaces as follows

$$
z=f(x)+g(y), y=f(x)+g(z), x=f(y)+g(z),
$$

we state three separate results.

Theorem 3.1. A $\nabla$-singular minimal translation surface in $\mathbb{R}^{3}$ of type $z=f(x)+g(y)$ with respect to a horizontal vector $\mathbf{u}$ is a generalized cylinder and one of the following occurs

(1) $f(x)=c_{1}$ and $g(y)=-\frac{1}{2} \ln \left|\cos \left(2 y+c_{2}\right)\right|+c_{3}$,

(2) $g(y)=c_{4}+c_{5} y$ and $f$ is a solution of the ordinary differential equation (ODE)

$$
f^{\prime \prime}=\frac{-\alpha}{\left(1+c_{5}^{2}\right) x}\left(f^{\prime}\right)^{3}+\frac{2}{1+c_{5}^{2}}\left(f^{\prime}\right)^{2}-\frac{\alpha}{x} f^{\prime}+2,
$$

where $c_{1}, \ldots, c_{5} \in \mathbb{R}$ and $f^{\prime}=\frac{d f}{d x}$, etc.

Proof. The unit normal vector field and mean curvature are computed by

$$
\xi=\frac{-f^{\prime} \partial_{x}-g^{\prime} \partial_{y}+\partial_{z}}{\sqrt{1+\left(f^{\prime}\right)^{2}+\left(g^{\prime}\right)^{2}}}
$$

and

$$
H^{\nabla}=\frac{\left[1+\left(g^{\prime}\right)^{2}\right] f^{\prime \prime}+\left[1+\left(f^{\prime}\right)^{2}\right] g^{\prime \prime}-2\left[1+\left(f^{\prime}\right)^{2}+\left(g^{\prime}\right)^{2}\right]}{2\left[1+\left(f^{\prime}\right)^{2}+\left(g^{\prime}\right)^{2}\right]^{\frac{3}{2}}},
$$

where $g^{\prime}=\frac{d g}{d y}$ and so. Without of loss of generality, we may assume that $\mathbf{u}=\partial_{x}$. Then Eq. (2.3) gives

$$
\frac{\left[1+\left(g^{\prime}\right)^{2}\right] f^{\prime \prime}+\left[1+\left(f^{\prime}\right)^{2}\right] g^{\prime \prime}-2\left[1+\left(f^{\prime}\right)^{2}+\left(g^{\prime}\right)^{2}\right]}{1+\left(f^{\prime}\right)^{2}+\left(g^{\prime}\right)^{2}}=-\alpha \frac{f^{\prime}}{x} .
$$

To solve Eq. (3.2), we distinguish several cases: the first case is that $f(x)=f_{0} \in \mathbb{R}$. Thus, Eq. (3.2) reduces to $g^{\prime \prime}=2\left(g^{\prime}\right)^{2}+2$. Solving this implies the first statement of the theorem. The second case is that $f^{\prime}=f_{0} \neq 0$. Hence, Eq. (3.2) reduces to the following polynomial equation on $x$

$$
\left\{g^{\prime \prime}\left(1+f_{0}^{2}\right)-2\left[1+f_{0}^{2}+\left(g^{\prime}\right)^{2}\right]\right\} x+\alpha f_{0}\left[1+f_{0}^{2}+\left(g^{\prime}\right)^{2}\right]=0,
$$

which means that this case is false because the constant term of the polynomial equation cannot be zero. The last case is that $f^{\prime \prime} \neq 0$. Then taking partial derivative Eq. (3.2) with respect to $y$ leads to

$$
2\left(-2+f^{\prime \prime}\right) g^{\prime} g^{\prime \prime}+\left[1+\left(f^{\prime}\right)^{2}\right] g^{\prime \prime \prime}=-2 \alpha g^{\prime} g^{\prime \prime} \frac{f^{\prime}}{x} \text {. }
$$

That $g^{\prime}=g_{0}, g_{0} \in \mathbb{R}$, is clearly a solution of Eq. (3.3). Then Eq. (3.2) reduces to

$$
\left[-2-2 g_{0}^{2}+\left(1+g_{0}^{2}\right) f^{\prime \prime}-2\left(f^{\prime}\right)^{2}\right] x+\alpha f^{\prime}\left[1+g_{0}^{2}+\left(f^{\prime}\right)^{2}\right]=0,
$$

or

$$
f^{\prime \prime}+\frac{\alpha}{\left(1+g_{0}^{2}\right) x}\left(f^{\prime}\right)^{3}-\frac{2}{1+g_{0}^{2}}\left(f^{\prime}\right)^{2}+\frac{\alpha}{x} f^{\prime}-2=0
$$

which gives the second statement of the theorem. Then the proof finishes if we show that Eq. (3.3) has no solution for $f^{\prime \prime} g^{\prime \prime} \neq 0$. By contradiction, assume that $f^{\prime \prime} g^{\prime \prime} \neq 0$. Dividing Eq. (3.3) with $2 g^{\prime} g^{\prime \prime}$, we get

$$
\left[1+\left(f^{\prime}\right)^{2}\right] \frac{g^{\prime \prime \prime}}{2 g^{\prime} g^{\prime \prime}}+f^{\prime \prime}+\alpha \frac{f^{\prime}}{x}-2=0
$$


yielding

Integrating Eq. (3.5) gives

$$
g^{\prime \prime \prime}=2 c g^{\prime} g^{\prime \prime}, c \in \mathbb{R}
$$

$$
g^{\prime \prime}=c\left(g^{\prime}\right)^{2}+d, d \in \mathbb{R}
$$

Substituting Eq. (3.6) to Eq. (3.2) leads to

$$
f^{\prime \prime}+\left[1+\left(f^{\prime}\right)^{2}\right]\left(-2+d+\alpha \frac{f^{\prime}}{x}\right)=0 .
$$

From Eqs (3.4) and (3.7), we conclude two equations

$$
f^{\prime \prime}=\frac{\left(-2+\alpha \frac{f^{\prime}}{x}\right)\left(-2+d+\alpha \frac{f^{\prime}}{x}\right)}{2+c-d-\alpha \frac{f^{\prime}}{x}}
$$

and

$$
(-2-c+d)\left(f^{\prime}\right)^{2}+\alpha \frac{\left(f^{\prime}\right)^{3}}{x}+d-c=0 .
$$

Notice that the denominator in Eq. (3.8) is not zero because the contradiction $f^{\prime \prime}=0$ is obtained otherwise. Taking derivative of Eq. (3.9) leads to

$$
\left[2(-2-c+d)+\frac{3 \alpha f^{\prime}}{x}\right] f^{\prime \prime}=\alpha\left(\frac{f^{\prime}}{x}\right)^{2}
$$

Considering Eq. (3.8) to Eq. (3.10) gives a polynomial equation of $\frac{f^{\prime}}{x}$

$$
\begin{gathered}
\alpha^{2}(1+3 \alpha)\left(\frac{f^{\prime}}{x}\right)^{3}+\alpha[\alpha(-16-2 c+5 d)-2-c+d]\left(\frac{f^{\prime}}{x}\right)^{2} \\
2 \alpha[(-4+d)(-2-c+d)-3(-2+d)] \frac{f^{\prime}}{x}-4(-2-c+d)(-2+d)=0,
\end{gathered}
$$

in which the fact that the leading coefficient vanish yields $\alpha=\frac{-1}{3}$ and therefore Eq. (3.11) reduces

$$
\begin{gathered}
\frac{-10+c+2 d}{9}\left(\frac{f^{\prime}}{x}\right)^{2}-\frac{2}{3}[(-4+d)(-2-c+d)-3(-2+d)] \frac{f^{\prime}}{x} \\
-4(-2-c+d)(-2+d)=0 .
\end{gathered}
$$

The constant term must be zero and for this reason assume that $d=2$. Then Eq. (3.12) gives the contradiction $c=6$ and $c=0$. This means that $d \neq 2$ and $-2-c+d=0$ and therefore the coefficient of the term of degree 1 cannot vanish, a contradiction.

The ODE (3.1) admits a reduction of order

$$
u^{\prime}=\frac{-\alpha}{\left(1+c_{5}^{2}\right) x} u^{3}+\frac{2}{1+c_{5}^{2}} u^{2}-\frac{\alpha}{x} u+2,
$$

where $u=u(x)=f^{\prime}(x)$ and $u^{\prime}(x)=f^{\prime \prime}(x)$. Due to $\alpha \neq 0$, the ODE (3.13) is an Abel equation of the first kind given by

$$
u^{\prime}=p_{3}(x) u^{3}+p_{2}(x) u^{2}+p_{1}(x) u+p_{0}(x)
$$

and no admits a general solution in terms of known functions, excepting very special cases depending on the functions $p_{0}(x), \ldots, p_{3}(x)$. We refer to $[46,47]$ for more details. Notice also that the surface given by the first statement of Theorem 3.1 is both $\nabla$-singular minimal and $\nabla$-minimal.

Theorem 3.2. A $\nabla$-singular minimal translation surface in $\mathbb{R}^{3}$ of type $y=f(x)+g(z)$ with respect to a horizontal vector $\mathbf{u}$ is either a plane parallel to $\mathbf{u}$ or a generalized cylinder satisfying one of the following

(1) $f(x)=c_{1}$ and

$$
g(z)= \pm \frac{1}{2} \arctan \left(\frac{1}{c_{2}} \sqrt{e^{4 z}-c_{2}^{2}}\right)+c_{3},
$$

(2) $g(z)=c_{4}+c_{5} z$ and $f$ is a solution of the $O D E$

$$
f^{\prime \prime}=\frac{-\alpha}{\left(1+c_{5}^{2}\right) x}\left(f^{\prime}\right)^{3}-\frac{2 c_{5}}{1+c_{5}^{2}}\left(f^{\prime}\right)^{2}-\frac{\alpha}{x} f^{\prime}-2 c_{5},
$$

where $c_{1}, \ldots, c_{5} \in \mathbb{R}, c_{2} \neq 0$, and $f^{\prime}=\frac{d f}{d x}$, etc. 
Proof. The unit normal vector field and mean curvature are given by

$$
\xi=\frac{f^{\prime} \partial_{x}-\partial_{y}+g^{\prime} \partial_{z}}{\sqrt{1+\left(f^{\prime}\right)^{2}+\left(g^{\prime}\right)^{2}}}
$$

and

$$
2 H^{\nabla}=-\frac{\left[1+\left(g^{\prime}\right)^{2}\right] f^{\prime \prime}+\left[1+\left(f^{\prime}\right)^{2}\right] g^{\prime \prime}+2\left[1+\left(f^{\prime}\right)^{2}+\left(g^{\prime}\right)^{2}\right] g^{\prime}}{\left[1+\left(f^{\prime}\right)^{2}+\left(g^{\prime}\right)^{2}\right]^{\frac{3}{2}}},
$$

where $g^{\prime}=\frac{d g}{d z}$ and so. Without of loss of generality we may assume that $\mathbf{u}=\partial_{x}$. Therefore, Eq. (2.3) implies

$$
\frac{f^{\prime \prime}\left[1+\left(g^{\prime}\right)^{2}\right]+g^{\prime \prime}\left[1+\left(f^{\prime}\right)^{2}\right]+2\left[1+\left(f^{\prime}\right)^{2}+\left(g^{\prime}\right)^{2}\right] g^{\prime}}{1+\left(f^{\prime}\right)^{2}+\left(g^{\prime}\right)^{2}}=-\alpha \frac{f^{\prime}}{x} .
$$

To solve Eq. (3.14), we distinguish several cases: the first case is that $f(x)=f_{0} \in \mathbb{R}$. Then, Eq. (3.14) reduces to $g^{\prime \prime}=-2\left[1+\left(g^{\prime}\right)^{2}\right] g^{\prime}$. That $g^{\prime}=0$ is a trivial solution of this equation and leads the surface to a plane parallel to $\mathbf{u}$. Otherwise, $g^{\prime} \neq 0$, after solving it, we obtain the first statement of the theorem. The second case is that $f^{\prime}=f_{0} \neq 0$. Then Eq. (3.14) reduces to a polynomial equation on

$$
\left\{\left[1+f_{0}^{2}\right] g^{\prime \prime}+2\left[1+f_{0}^{2}+\left(g^{\prime}\right)^{2}\right] g^{\prime}\right\} x+\alpha f_{0}\left(1+f_{0}^{2}+\left(g^{\prime}\right)^{2}\right)=0
$$

which implies that this case is false because the constant term of the polynomial equation cannot be zero. The last case is that $f^{\prime \prime} \neq 0$. Taking partial derivative Eq. (3.14) with respect to $z$, we get

$$
6\left(g^{\prime}\right)^{2} g^{\prime \prime}+2\left(f^{\prime \prime}+\alpha \frac{f^{\prime}}{x}\right) g^{\prime} g^{\prime \prime}+\left[1+\left(f^{\prime}\right)^{2}\right]\left(2 g^{\prime \prime}+g^{\prime \prime \prime}\right)=0 .
$$

That $g^{\prime}=g_{0} \in \mathbb{R}$ is clearly a solution to Eq. (3.15). So, Eq. (3.14) reduces to

$$
f^{\prime \prime}+\frac{\alpha}{\left(1+g_{0}^{2}\right) x}\left(f^{\prime}\right)^{3}+\frac{2 g_{0}}{1+g_{0}^{2}}\left(f^{\prime}\right)^{2}+\frac{\alpha}{x} f^{\prime}+2 g_{0}=0,
$$

which gives the second statement of the theorem. Then the proof finishes if we show that Eq. (3.15) has no solution for $f^{\prime \prime} g^{\prime \prime} \neq 0$. By contradiction, assume that $f^{\prime \prime} g^{\prime \prime} \neq 0$. Dividing Eq. (3.15) with $2 g^{\prime} g^{\prime \prime}$, we have

$$
\left(\frac{g^{\prime \prime \prime}}{2 g^{\prime} g^{\prime \prime}}+\frac{1}{g^{\prime}}\right)\left[1+\left(f^{\prime}\right)^{2}\right]+f^{\prime \prime}+\alpha \frac{f^{\prime}}{x}+3 g^{\prime}=0
$$

Taking partial derivative of Eq. (3.16) with respect to $x$ and $z$ leads to

$$
f^{\prime} f^{\prime \prime}\left(\frac{g^{\prime \prime \prime}}{2 g^{\prime} g^{\prime \prime}}+\frac{1}{g^{\prime}}\right)^{\prime}=0
$$

or, owing to $f^{\prime} f^{\prime \prime} \neq 0$, the term $g^{\prime \prime \prime} /\left(2 g^{\prime} g^{\prime \prime}\right)+1 / g^{\prime}$ becomes a constant. Therefore, the partial derivative of Eq. (3.16) with respect to $z$ gives $g^{\prime \prime}=0$, a contradiction

Note that, as in previous result, the surface given by the first statement of Theorem 3.2 is both $\nabla$-singular minimal and $\nabla$-minimal.

Theorem 3.3. A $\nabla$-singular minimal translation surface in $\mathbb{R}^{3}$ of type $x=f(y)+g(z)$ with respect to a horizontal vector $\mathbf{u}$ is a generalized cylinder and one of the following occurs

(1) $f(y)=c_{1}$ and $g$ is a solution of the autonomous $O D E$

$$
g^{\prime \prime}=\left(\frac{\alpha}{c_{1}+g}-2 g^{\prime}\right)\left[1+\left(g^{\prime}\right)^{2}\right], g^{\prime \prime} \neq 0
$$

(2) $g(z)=c_{2}$ and

$$
y= \pm \int\left[c_{3}\left(f+c_{2}\right)^{2 \alpha}+c_{4}\right]^{-1 / 2} d f
$$

for $c_{1}, \ldots, c_{4} \in \mathbb{R}, c_{3} \neq 0$, and $g^{\prime}=\frac{d g}{d z}$, etc. 
Proof. The unit normal vector field and mean curvature are

and

$$
\xi=\frac{\left(\partial_{x}-f^{\prime} \partial_{y}-g^{\prime} \partial_{z}\right)}{\sqrt{1+\left(f^{\prime}\right)^{2}+\left(g^{\prime}\right)^{2}}}
$$

$$
2 H^{\nabla}=\frac{\left[1+\left(g^{\prime}\right)^{2}\right] f^{\prime \prime}+\left[1+\left(f^{\prime}\right)^{2}\right] g^{\prime \prime}+2\left[1+\left(f^{\prime}\right)^{2}+\left(g^{\prime}\right)^{2}\right] g^{\prime}}{\left[1+\left(f^{\prime}\right)^{2}+\left(g^{\prime}\right)^{2}\right]^{\frac{3}{2}}},
$$

where $f^{\prime}=\frac{d f}{d y}$ and so. Without of loss of generality we may assume that $\mathbf{u}=\partial_{x}$. Hence Eq. (2.3) turns to

$$
\frac{\left[1+\left(g^{\prime}\right)^{2}\right] f^{\prime \prime}+\left[1+\left(f^{\prime}\right)^{2}\right] g^{\prime \prime}+2\left[1+\left(f^{\prime}\right)^{2}+\left(g^{\prime}\right)^{2}\right] g^{\prime}}{1+\left(f^{\prime}\right)^{2}+\left(g^{\prime}\right)^{2}}=\alpha \frac{1}{f+g},
$$

in which both $f$ and $g$ cannot be constant simultaneously because the situation $\alpha=0$ is obtained otherwise. We distinguish the remaining cases: the first case is that $f(y)=f_{0} \in \mathbb{R}$. Eq. (3.17) writes

$$
g^{\prime \prime}+2\left[1+\left(g^{\prime}\right)^{2}\right] g^{\prime}=\alpha \frac{1+\left(g^{\prime}\right)^{2}}{f_{0}+g} .
$$

in which $g$ must be non-linear because the situation $\alpha=0$ is obtained otherwise. This concludes the first statement of the theorem. The second case is that $f(y)=d+c y, c, d \in \mathbb{R}, c \neq 0$. Then Eq. (3.17) turns to

$$
\frac{\left(1+c^{2}\right) g^{\prime \prime}}{1+c^{2}+\left(g^{\prime}\right)^{2}}+2 g^{\prime}=\alpha \frac{1}{d+c y+g}
$$

By taking partial derivative of Eq. (3.18) with respect to $y$, we obtain the contradiction $\alpha c=0$. The third case is that $f^{\prime \prime} \neq 0$ and $g=g_{0} \in \mathbb{R}$. Then Eq. (3.17) reduces to

$$
\frac{f^{\prime \prime}}{1+\left(f^{\prime}\right)^{2}}=\alpha \frac{1}{f+g_{0}} \text {. }
$$

By multiplying Eq. (3.19) with $2 f^{\prime}$ and taking first integral, we obtain

$$
f^{\prime}= \pm \sqrt{c\left(f+g_{0}\right)^{2 \alpha}-1}, c \in \mathbb{R}, c \neq 0 .
$$

Taking derivative of Eq. (3.20), we can conclude

$$
f^{\prime \prime}=\alpha c\left(f+g_{0}\right)^{2 \alpha-1},
$$

which is known as Emden-Fowler equation (see [47]) and the solution follows

$$
y= \pm \int\left[c\left(f+g_{0}\right)^{2 \alpha}+d\right]^{-1 / 2} d f+e, d, e \in \mathbb{R},
$$

which proves the second statement of the theorem. The fourth case is that $f^{\prime \prime} \neq 0$ and $g(z)=d+c z, c, d \in \mathbb{R}, c \neq 0$. Then Eq. (3.17) reduces to

$$
\frac{\left[1+c^{2}\right] f^{\prime \prime}}{1+c^{2}+\left(f^{\prime}\right)^{2}}+2 c=\alpha \frac{1}{d+c z+f}
$$

By taking partial derivative of Eq. (3.21) with respect to $z$, we obtain the contradiction $\alpha c=0$. Therefore the proof finishes if we show that Eq. (3.17) has no solution for $f^{\prime \prime} g^{\prime \prime} \neq 0$. By contradiction, assume that $f^{\prime \prime} g^{\prime \prime} \neq 0$. Then Eq. (3.17) can be rewritten as

$$
\begin{gathered}
(f+g)\left\{\left[1+\left(g^{\prime}\right)^{2}\right] f^{\prime \prime}+\left[1+\left(f^{\prime}\right)^{2}\right] g^{\prime \prime}+2\left[1+\left(f^{\prime}\right)^{2}+\left(g^{\prime}\right)^{2}\right] g^{\prime}\right\} \\
=\alpha\left[1+\left(f^{\prime}\right)^{2}+\left(g^{\prime}\right)^{2}\right] .
\end{gathered}
$$

If we take partial derivative of Eq. (3.22) with respect to $y$ and $z$ and afterwards divide the generated equation with $f^{\prime} f^{\prime \prime} g^{\prime} g^{\prime \prime}$, we can deduce

$$
\begin{gathered}
4+\frac{f^{\prime \prime \prime}}{f^{\prime} f^{\prime \prime}}\left[\frac{1+\left(g^{\prime}\right)^{2}}{g^{\prime \prime \prime}}+2 g\right]+\left(\frac{g^{\prime \prime \prime}}{g^{\prime} g^{\prime \prime}}+\frac{2}{g^{\prime}}\right)\left[\frac{1+\left(f^{\prime}\right)^{2}}{f^{\prime \prime}}+2 f\right] \\
+\frac{2 f f^{\prime \prime \prime}}{f^{\prime} f^{\prime \prime}}+\frac{2 g g^{\prime \prime \prime}}{g^{\prime} g^{\prime \prime}}+\frac{4 g}{g^{\prime}}+\frac{4 g^{\prime}}{g^{\prime \prime}}+\frac{6 g^{\prime}}{f^{\prime \prime}}=0 .
\end{gathered}
$$


The partial derivative of Eq. (3.23) with respect to $y$ and $z$ leads to

We have subcases:

$$
\left(\frac{f^{\prime \prime \prime}}{f^{\prime} f^{\prime \prime}}\right)^{\prime}\left[\frac{1+\left(g^{\prime}\right)^{2}}{g^{\prime \prime}}+2 g\right]^{\prime}+\left(\frac{g^{\prime \prime \prime}}{g^{\prime} g^{\prime \prime}}+\frac{2}{g^{\prime}}\right)^{\prime}\left[\frac{1+\left(f^{\prime}\right)^{2}}{f^{\prime \prime}}+2 f\right]^{\prime}-\frac{6 g^{\prime \prime} f^{\prime \prime \prime}}{\left(f^{\prime \prime}\right)^{2}}=0 .
$$

(1) $f^{\prime \prime}=f_{0} \in \mathbb{R}, f_{0} \neq 0$. Then the partial derivative of Eq. (3.23) with respect to $y$ leads to

$$
\frac{g^{\prime \prime \prime}}{g^{\prime} g^{\prime \prime}}+\frac{2}{g^{\prime}}=0
$$

Therefore Eq. (3.23) reduces to

$$
2+\frac{2 g^{\prime}}{g^{\prime \prime}}+\frac{3 g^{\prime}}{f_{0}}=0
$$

On the other hand, a first integration of Eq. (3.25) gives

$$
g^{\prime \prime}=-2 g^{\prime}+c, c \in \mathbb{R} \text {. }
$$

By substituting Eq. (3.27) into Eq. (3.26), we obtain a polynomial equation on $g^{\prime}$ whose leading coefficient coming from the term $\left(g^{\prime}\right)^{2}$ is $\frac{-6}{f_{0}}$, a contradiction.

(2) $f^{\prime \prime \prime}=2 c f^{\prime} f^{\prime \prime}, c \in \mathbb{R}, c \neq 0$. A first integration yields $f^{\prime \prime}=c\left(f^{\prime}\right)^{2}+d, d \in \mathbb{R}$. Then Eq. (3.24) reduces to

$$
\left(\frac{g^{\prime \prime \prime}}{g^{\prime} g^{\prime \prime}}+\frac{2}{g^{\prime}}\right)^{\prime}\left[\frac{2 d}{c}-1+\left(f^{\prime}\right)^{2}\right]-6 g^{\prime \prime}=0
$$

which implies $\left(\frac{g^{\prime \prime \prime}}{g^{\prime} g^{\prime \prime}}+\frac{2}{g^{\prime}}\right)^{\prime}=0$. This gives from Eq. (3.24) the contradiction $g^{\prime \prime} f^{\prime \prime \prime}=0$.

(3) $\left(f^{\prime \prime \prime} / f^{\prime} f^{\prime \prime}\right)^{\prime} \neq 0$. Eq. (3.24) can be rewritten by dividing $g^{\prime \prime}\left(f^{\prime \prime \prime} / f^{\prime} f^{\prime \prime}\right)^{\prime}$ as

$$
A(y) B(z)=C(y)+D(z)
$$

where

and

$$
A(y)=\frac{\left[\left\{1+\left(f^{\prime}\right)^{2}\right\} / f^{\prime \prime}+2 f\right]^{\prime}}{\left(f^{\prime \prime \prime} / f^{\prime} f^{\prime \prime}\right)^{\prime}}, B(z)=\left(g^{\prime \prime \prime} / g^{\prime} g^{\prime \prime}+2 / g^{\prime}\right)^{\prime} / g^{\prime \prime}
$$

$$
C(y)=6 \frac{f^{\prime \prime \prime} /\left(f^{\prime \prime}\right)^{2}}{\left(f^{\prime \prime \prime} / f^{\prime} f^{\prime \prime}\right)^{\prime}}, D(z)=-\left[\left\{1+\left(g^{\prime}\right)^{2}\right\} / g^{\prime \prime}+2 g\right]^{\prime} / g^{\prime \prime} .
$$

The functions $A, B, C, D$ from Eq. (3.28) must be all constant. Let us put $B(z)=B_{0}$ and $D(z)=-D_{0}$, $B_{0}, D_{0} \in \mathbb{R}$. Therefore, we get

and

$$
\left[\frac{g^{\prime \prime \prime}}{g^{\prime} g^{\prime \prime}}+\frac{2}{g^{\prime}}\right]^{\prime}=B_{0} g^{\prime \prime}
$$

A first integration of Eq. (3.29) yields

$$
4 g^{\prime}-\left[1+\left(g^{\prime}\right)^{2}\right] \frac{g^{\prime \prime \prime}}{\left(g^{\prime \prime}\right)^{2}}=D_{0} g^{\prime \prime}
$$

$$
\frac{g^{\prime \prime \prime}}{g^{\prime} g^{\prime \prime}}+\frac{2}{g^{\prime}}=B_{0} g^{\prime}+d_{1}
$$

for $d_{1} \in \mathbb{R}$. Multiplying Eq. (3.31) with $g^{\prime} g^{\prime \prime}$ and then taking first integration the generated equation gives

$$
g^{\prime \prime}=\frac{B_{0}}{3}\left(g^{\prime}\right)^{3}+\frac{d_{1}}{2}\left(g^{\prime}\right)^{2}-2 g^{\prime}+d_{2},
$$

for $d_{2} \in \mathbb{R}$. By considering Eq. (3.32) into Eq. (3.31), we deduce that

$$
g^{\prime \prime \prime}=\frac{B_{0}^{2}}{3}\left(g^{\prime}\right)^{5}+\text { rest terms. }
$$

Substituting Eqs. (3.32) and (3.33) into Eq. (3.30), we have a polynomial equation on $g^{\prime}$ whose coefficient coming from $\left(g^{\prime}\right)^{7}$ is $\frac{B_{0}^{2}}{9}$, yielding $B_{0}=0$. It follows from Eq. (3.31)

$$
g^{\prime \prime \prime}=\left(-2+d_{1} g^{\prime}\right) g^{\prime \prime}
$$


and plugging it into Eq. (3.30)

$$
4 g^{\prime} g^{\prime \prime}-\left[1+\left(g^{\prime}\right)^{2}\right]\left(-2+d_{1} g^{\prime}\right)=D_{0}\left(g^{\prime \prime}\right)^{2} .
$$

Considering Eq. (3.32) into Eq. (3.34) leads to a polynomial equation on $g^{\prime}$ whose coefficient coming from $\left(g^{\prime}\right)^{3}$ is $d_{1}$ which must vanish. Therefore Eqs. (3.32) and (3.34) reduce to

$$
g^{\prime \prime}=-2 g^{\prime}+d_{2}
$$

and

$$
4 g^{\prime} g^{\prime \prime}+2\left(g^{\prime}\right)^{2}+2=D_{0}\left(g^{\prime \prime}\right)^{2},
$$

respectively. From these two equations, we can conclude a polynomial equation on $g^{\prime}$

$$
\left(4 D_{0}+6\right)\left(g^{\prime}\right)^{2}-4\left(D_{0}+d_{2}\right) g^{\prime}+D_{0} d_{2}^{2}-2=0,
$$

which gives a contradiction because the constant term cannot vanish.

\section{D-singular Minimal Translation Surfaces}

As in previous section, we characterize translation surfaces in $\mathbb{R}^{3}$ of each type to be $D$-singular minimal through the following results.

Theorem 4.1. A $D$-singular minimal translation surface in $\mathbb{R}^{3}$ of type $z=f(x)+g(y)$ with respect to a horizontal vector $\mathbf{u}$ is either a plane parallel to $\mathbf{u}$ or a generalized cylinder satisfying $g(y)=c_{1}+c_{2} y$ and

$$
f(x)= \pm\left|c_{3}\right| \sqrt{1+c_{2}^{2}} \int\left(x^{2 \alpha}-c_{3}^{2}\right)^{-1 / 2} d x
$$

where $c_{1}, c_{2}, c_{3} \in \mathbb{R}, c_{3} \neq 0$.

Proof. The unit normal vector field and mean curvature follow

$$
\xi=\frac{-f^{\prime} \partial_{x}-g^{\prime} \partial_{y}+\partial_{z}}{\sqrt{1+\left(f^{\prime}\right)^{2}+\left(g^{\prime}\right)^{2}}}
$$

and

$$
H^{D}=\frac{f^{\prime \prime}\left[1+\left(g^{\prime}\right)^{2}\right]+g^{\prime \prime}\left[1+\left(f^{\prime}\right)^{2}\right]}{2\left[1+\left(f^{\prime}\right)^{2}+\left(g^{\prime}\right)^{2}\right]^{\frac{3}{2}}},
$$

where $f^{\prime}=\frac{d f}{d x}, g^{\prime}=\frac{d g}{d y}$ and so. Without of loss of generality we may assume that $\mathbf{u}=\partial_{x}$. Hence Eq. (2.4) turns to

$$
\frac{\left[1+\left(g^{\prime}\right)^{2}\right] f^{\prime \prime}+\left[1+\left(f^{\prime}\right)^{2}\right] g^{\prime \prime}}{1+\left(f^{\prime}\right)^{2}+\left(g^{\prime}\right)^{2}}=-\alpha \frac{f^{\prime}}{x} .
$$

Eq. (4.1) has no solution if $g^{\prime \prime} \neq 0$, see [37, Theorem 5]. Therefore, we have $g^{\prime}=g_{0} \in \mathbb{R}$ and Eq. (4.1) reduces to

$$
\frac{\left(1+g_{0}^{2}\right) f^{\prime \prime}}{f^{\prime}\left[1+g_{0}^{2}+\left(f^{\prime}\right)^{2}\right]}=-\frac{\alpha}{x},
$$

where being $f$ a constant is a trivial solution, implying $M$ is a plane parallel to the vector $\mathbf{u}$. Assume that $f$ is no constant and then we can easily infer from Eq. (4.2) that $f$ cannot be a linear function, i.e. $f^{\prime \prime}=0$, due to $\alpha \neq 0$. Hence, a first integration of Eq. (4.2) yields

or

$$
\frac{f^{\prime}}{\sqrt{1+g_{0}^{2}+\left(f^{\prime}\right)^{2}}}=c x^{-\alpha}, c \in \mathbb{R}, c \neq 0,
$$

A first integration of Eq. (4.3) completes the proof.

$$
f^{\prime}= \pm|c| \frac{\sqrt{1+g_{0}^{2}}}{\sqrt{x^{2 \alpha}-c^{2}}} .
$$


For a translation surface of type $y=f(x)+g(z)$, the unit normal vector field and mean curvature follow

and

$$
\xi=\frac{f^{\prime} \partial_{x}-\partial_{y}+g^{\prime} \partial_{z}}{\sqrt{1+\left(f^{\prime}\right)^{2}+\left(g^{\prime}\right)^{2}}}
$$

$$
H^{D}=\frac{f^{\prime \prime}\left[1+\left(g^{\prime}\right)^{2}\right]+g^{\prime \prime}\left[1+\left(f^{\prime}\right)^{2}\right]}{2\left[1+\left(f^{\prime}\right)^{2}+\left(g^{\prime}\right)^{2}\right]^{\frac{3}{2}}},
$$

where $f^{\prime}=\frac{d f}{d x}, g^{\prime}=\frac{d g}{d z}$ and so. Then, with respect to the horizontal vector $\mathbf{u}=\partial_{x}$, the $D$-singular minimality equation is similar to Eq. (4.1) up to a sign. Thereby, for such a surface, we can state a similar result to Theorem 4.1 without proof by replacing $\alpha$ with $-\alpha$.

Theorem 4.2. A $D$-singular minimal translation surface in $\mathbb{R}^{3}$ of type $y=f(x)+g(z)$ with respect to a horizontal vector $\mathbf{u}$ is either a plane parallel to $\mathbf{u}$ or a generalized cylinder satisfying $g(z)=c_{1}+c_{2} z$ and

$$
f(x)= \pm\left|c_{3}\right| \sqrt{1+c_{2}^{2}} \int x^{\alpha}\left(1-c_{3}^{2} x^{2 \alpha}\right)^{-1 / 2} d x,
$$

where $c_{1}, c_{2}, c_{3} \in \mathbb{R}, c_{3} \neq 0$.

Theorem 4.3. A D-singular minimal translation surface in $\mathbb{R}^{3}$ of type $x=f(y)+g(z)$ with respect to a horizontal vector $\mathbf{u}$ is a generalized cylinder satisfying $f(y)=c_{1}$ and

$$
z= \pm \int\left[c_{2}^{2}\left(c_{1}+g\right)^{2 \alpha}-1\right]^{-1 / 2} d g
$$

for $c_{1}, c_{2} \in \mathbb{R}, c_{2} \neq 0$.

Proof. Without of loss of generality we may assume that $\mathbf{u}=\partial_{x}$. Then Eq. (2.4) implies

$$
\frac{\left[1+\left(g^{\prime}\right)^{2}\right] f^{\prime \prime}+\left[1+\left(f^{\prime}\right)^{2}\right] g^{\prime \prime}}{1+\left(f^{\prime}\right)^{2}+\left(g^{\prime}\right)^{2}}=\frac{\alpha}{f+g},
$$

in which the roles of $f$ and $g$ are symmetric. Eq. (4.4) has a solution provided that $f$ or $g$ is a constant, see [35, Theorem 4.1]. Thanks to the symmetry, we can assume $f=f_{0} \in \mathbb{R}$. Thereby, Eq. (4.5) reduces to

$$
\frac{g^{\prime \prime}}{1+\left(g^{\prime}\right)^{2}}=\frac{\alpha}{f_{0}+g} \text {. }
$$

Put

$$
g^{\prime}=q, q^{\prime}=\frac{d q}{d g} \frac{d g}{d z}=\frac{g^{\prime \prime}}{g^{\prime}}, q=q(g) .
$$

By considering Eq. (4.7) into Eq. (4.6) we get

$$
\frac{q q^{\prime}}{1+q^{2}}=\frac{\alpha}{f_{0}+g}
$$

A first integration of Eq. (4.8) with respect to $g$ yields $1+q^{2}=c^{2}\left(f_{0}+g\right)^{2 \alpha}$ or

$$
d z= \pm \frac{d g}{\sqrt{c^{2}\left(f_{0}+g\right)^{2 \alpha}-1}}
$$

and the proof is completed by a first integration.

\section{Conclusions}

In this study, new perspectives on the singular minimality of immersed surfaces in $\mathbb{R}^{3}$ were introduced. These, in particular the $\nabla$-singular minimality, provided us non-trivial and different results from the obtained one with respect to the Levi-Civita connection on $\mathbb{R}^{3}$, see [35,37]. Our results were found with respect to a horizontal vector and it will not make big difference when the vector is assumed to be vertical. Yet, the problem of finding $\nabla$ - and $D$-singular minimal translation surfaces in $\mathbb{R}^{3}$ with respect to an arbitrary vector is open. 


\section{CONFLICTS OF INTEREST}

The authors declare that there are no conflicts of interest regarding the publication of this article.

\section{Authors Contributions Statement}

All authors have contributed sufficiently in the planning, execution, or analysis of this study to be included as authors. All authors have read and agreed to the published version of the manuscript.

\section{REFERENCES}

[1] Agashe, N. S., Chafle, M.R., A semi-symmetric non-metric connection on a Riemannian manifold, Indian J. Pure Appl. Math. 23(6)(1992), 399-409.

[2] Agashe, N. S., Chafle, M.R., On submanifolds of a Riemannian manifold with a semi-symmetric non-metric connection, Tensor 55(2)(1994), $120-130$.

[3] Akyol, M. A., Beyendi, S., Riemannian submersion endowed with a semi-symmetric non-metric connection, Konuralp J. Math. 6(1)(2018), 188-193.

[4] Aydin, M.E., Erdur, A., Ergut, M., Singular minimal translation graphs in Euclidean spaces, J. Korean Math. Soc. 58(1)(2021), 109-122.

[5] Aydin, M.E., Mihai, A., Translation hypersurfaces and Tzitzeica Translation hyper-surfaces of the Euclidean space, Proc. Rom. Acad. Ser. A, Math. Phys. Tech. Sci. Inf. Sci. 16(4)(2015), 477-483.

[6] Böhme, R., Hildebrant, S., Taush, E., The two-dimensional analogue of the catenary, Pac. J. Math. 88(2)(1980), 247-278.

[7] Chaubey, S.K., Yildiz, A., Riemannian manifolds admitting a new type of semisymmetric nonmetric connection, Turk. J. Math. 43(4)(2019), $1887-1904$.

[8] Darboux, J.G., Théorie Génerale des Surfaces, Livre I, Gauthier-Villars, Paris, 1914.

[9] De, U.C., Barman, A., On a type of semisymmetric metric connection on a Riemannian manifold, Publ. Inst. Math., Nouv. Sér. 98(112)(2015), 211-218.

[10] Dierkes, U., A Bernstein result for enery minimizing hypersurfaces, Cal. Var. Part. Differ. Equ. 1(1)(1993), 37-54.

[11] Dierkes, U., Singular minimal surfaces, Geometric Analysis and Nonlinear Partial Differential Equations, Springer, Berlin, Heidelberg (2003), 176-193.

[12] Dillen, F., Van de Woestyne, I., Verstraelen, L., Walrave, J.T., The surface of Scherk in E3: A special case in the class of minimal surfaces defined as the sum of two curves, Bull. Inst. Math. Acad. Sin. (N.S.) 26(4)(1998), 257-267.

[13] Dillen, F., Verstraelen, L., Zafindratafa, G., A generalization of the translation surfaces of Scherk, Diff. Geom. in honor of Radu Rosca (KUL) (1991), 107-109.

[14] Dillen, F., Goemans, W., Van de Woestyne, I., Translation surfaces of Weingarten type in 3-space, Bull. Transilv. Univ. Braşov, Ser. III 1(50) (2008), 109-122.

[15] Dogru, Y., On some properties of submanifolds of a Riemannian manifold endowed with a semi-symmetric non-metric connection, An. Şt. Univ. Ovidius Constanta, 19(3)(2011), 85-100.

[16] Gil, J.B., The catenary (almost) everywhere, Boletìn de la AMV XII(2)(2005), 251-258.

[17] Goemans, W., Van de Woestyne, I., Translation and homothetical lightlike hypersurfaces of semi-Euclidean space, Kuwait J. Sci. Eng. 38(2)(2011), 35-42.

[18] Gozutok, A., Esin, E., Tangent bundle of hypersurface with semi symmetric metric connection, Int. J. Contemp. Math. Sci. 7(6)(2012), $279-289$.

[19] Gray, A., Modern Differential Geometry of Curves and Surfaces with Mathematica, CRC Press, 2nd Ed., 1998.

[20] Hasanis, T., Translation surfaces with non-zero constant mean curvature in Euclidean space, J. Geom. 110(20)(2019).

[21] Hasanis, T., López, R., Translation surfaces in Euclidean space with constant Gaussian curvature, Arxiv 8 Sept 2018: https://arxiv.org/abs/1809.02758v1.

[22] Hasanis, T., López, R., Classification and construction of minimal translation surfaces in Euclidean space, Result Math. 75(1)(2020), 1-22.

[23] Hayden, H. A., Subspaces of a space with torsion, Proc. London Math. Soc. S2-34(1)(1932), 27-50.

[24] Imai, T., Notes on semi-symmetric metric connections, Tensor 24(1972), 293-296.

[25] Inoguchi, J., López, R., Munteanu, M.I., Minimal translation surfaces in the Heisenberg group Nil, Geom. Dedicata 161(2012), $221-231$.

[26] Jung, S.D., Liu, H., Liu, Y., Weingarten affine translation surfaces in Euclidean 3-space, Results Math. 72(4)(2017),1839-1848.

[27] Lima, B.P., Santos, N. L., Sousa, P.A., Generalized translation hypersurfaces in Euclidean space, J. Math. Anal. Appl. 470(2)(2019), 11291135.

[28] Liu, H., Translation surfaces with constant mean curvature in 3- dimensional spaces, J. Geom. 64(1999), 141-149.

[29] Liu, H., Jung, S.D., Affine translation surfaces with constant mean curvature in Euclidean 3-space, J. Geom. 108(2017), 423-428.

[30] Liu, H., Yu, Y., Affine translation surfaces in Euclidean 3-space, Proc. Japan Acad. 89(A)(2013), 111-113.

[31] López, R., Minimal translation surfaces in hyperbolic space, Beitr. Algebra Geom. 52(1)(2011), 105-112.

[32] López, R., Munteanu, M.I., Minimal translation surfaces in Sol 3 , J. Math. Soc. Japan 64(3)(2012), 985-1003.

[33] López, R., Moruz, M., Translation and homothetical surfaces in Euclidean space with constant curvature, J. Korean Math. Soc. 52(3)(2015), 523-535.

[34] López, R., Constant Mean Curvature Surfaces with Boundary, Springer-Verlag, Berlin, 2013.

[35] López, R., Seperation of variables in equations of mean curvature type, Poc. R. Soc. Edinb. 146(5)(2016), 1017-1035.

[36] López, R., Perdomo, O., Minimal translation surfaces in Euclidean space, J. Geom. Anal. 27(4)(2017), 2926-2937. 
[37] López, R., Invariant singular minimal surfaces, Ann. Glob. Anal. Geom. 53(4)(2018), 521-541.

[38] López, R., The Dirichlet problem for the $\alpha$-singular minimal surface equation, Arch. Math. 112(2)(2019), $213-222$.

[39] López, R., The two-dimensional analogue of the Lorentzian catenary and the Dirichlet problem, Pacific J. Math. 305(2)(2020), 693-719.

[40] Moruz, M., Munteanu, M.I., Minimal translation hypersurfaces in $\mathbb{E}^{4}$, J. Math. Anal. Appl. 439(2)(2016), $798-812$.

[41] Munteanu, M.I., Palmas, O., Ruiz-Hernandez, G., Minimal translation hypersurfaces in Euclidean spaces, Mediterran. J. Math. 13(5)(2016), 2659-2676.

[42] Murathan, C., Ozgür, C., Riemannian manifolds with a semi-symmetric metric connection satisfying some semisymmetry conditions, Proc. Est. Acad. Sci. 57(4)(2008), 210-216.

[43] Nakao, Z., Submanifolds of a Riemannian manifold with semisymmetric metric connections, Proc. Amer. Math. Soc. 54(1)(1976), 261-266.

[44] Ozen Zengin, F., Altay Demirbag, S., Uysal, S. A., Some vector fields on a Riemannian manifold with semi-symmetric metric connection, Bull. Iranian Math. Soc. 38(2)(2012), 479-490.

[45] Ozgur, C., On submanifolds of a Riemannian manifold with a semi-symmetric non-metric connection, Kuwait J. Sci. Eng. 37(2)(2010), 17-30.

[46] Panayotounakos, D.E., Zarmpoutis, T.I., Construction of exact parametric or closed form solutions of some unsolvable classes of nonlinear ODEs (Abel's nonlinear ODEs of the first kind and relative degenerate equations), Int. J. Math. Math. Sci. (2011), p. 387429.

[47] Polyanin, A.D., Zaitsev, V.F., Handbook of Exact Solutions for Ordinary Differential Equations, Chapman \& Hall/CRC, Boca Raton, Fla, USA, 2nd Ed., 2003.

[48] Scherk, H.F., Bemerkungen über die kleinste Fläche innerhalb gegebener Grenzen, J. Reine Angew. Math. 13(1835), $185-208$.

[49] Sekerci, G.A., Sevinc, S., Coken, A.C., On the translation hypersurfaces with Gauss map Gatisfying $\triangle G=A G$, Miscolc Math. Notes 20(2)(2019), 1215-1225.

[50] Seo, K., Translation hypersurfaces with constant curvature in space form, Osaka J. Math. 50(3)(2013), 631-641.

[51] Sun, H., On affine translation surfaces of constant mean curvature, Kumamoto J. Math. 13(2000), 49-57.

[52] Unal, I., On submanifolds of $N(k)$-quasi Einstein manifolds with a type of semi-symmetric metric connection, Univers. J. Math. Appl. 3(4)(2020), 167-172.

[53] Verstraelen, L., Walrave, J., Yaprak, S., The minimal translation surfaces in Euclidean space, Soochow J. Math. 20(1)(1994), 77-82.

[54] Wang, Y., Minimal translation surfaces with respect to semi-symmetric connections in $\mathbb{R}^{3}$ and $\mathbb{R}_{1}^{3}$, Bull. Korean Math. Soc. 58(4)(2021), 959-972.

[55] Yang, D., Fu, Y.,On affine translation surfaces in affine space, J. Math. Anal. Appl. 440(2)(2016), $437-450$.

[56] Yang, D., Zhang, J., Fu, Y., A note on minimal translation graphs in Euclidean space, Mathematics 7(10)(2019).

[57] Yano, K., On semi-symmetric metric connection, Rev. Roumaine Math. Pures Appl. 15(1970), $1579-1586$.

[58] Yano, K., Kon, M., Structures on Manifolds, Series in Pure Math., World Scientific, 1984.

[59] Yoon, D.W., On the Gauss map of translation surfaces in Minkowski 3-space, Taiwanese J. Math. 6(2002), 389-398.

[60] Yoon, D.W., Lee, C.W., Karacan, M.K., Some translation surfaces in the 3-dimensional Heisenberg group, Bull. Korean Math. Soc. 50(4)(2013), 1329-1343.

[61] Yucesan, A., Yasar, E., Non-degenerate hypersurfaces of a semi-Riemannian manifold with a semi-symmetric non-metric connection, Math. Rep. (Bucur) 14(64) (2012), no. 2, 209-219. 